Int. J. Mol. Sci. 2014, 15, 11973-11983; doi:10.3390/ijms150711973

OPEN ACCESS

International Journal of

Molecular Sciences

ISSN 1422-0067

www.mdpi.com/journal/ijms

Article

\title{
miR-143 Inhibits NSCLC Cell Growth and Metastasis by Targeting Limk1
}

Hui Xia ${ }^{1, \dagger}$, Shengjie Sun ${ }^{2, \dagger}$, Bo Wang ${ }^{3}$, Tao Wang ${ }^{3}$, Chaoyang Liang ${ }^{3}$, Guo Li ${ }^{3}$, Chongbiao Huang ${ }^{4}$, Daliang $\mathrm{Qi}^{4, *}$ and Xiangyang Chu ${ }^{3, *}$

1 Department of Thoracic-Cardio Surgery, the First Affiliated Hospital of PLA General Hospital, Beijing 100048, China; E-Mail: hui_xiaa@126.com

2 Department of Medical Oncology, the General Hospital of People's Liberation Army, Beijing 100853, China; E-Mail: shengjie_sunn@126.com

3 Department of Thoracic Surgery, the General Hospital of People's Liberation Army, Beijing 100853, China; E-Mails: wang_boa@126.com (B.W.); wang_taow@126.com (T.W.); liang_cyc@126.com (C.L.); li_gguo@126.com (G.L.)

4 Key Laboratory of Cancer Prevention and Therapy of Tianjin, Department of Senior Ward, Tianjin Medical University Cancer Institute and Hospital, Huanhu Xilu, Hexi District, Tianjin 300060, China; E-Mail: huang_chongbiao@126.com

$\dagger$ These authors contributed equally to this work.

* Authors to whom correspondence should be addressed;

E-Mails: chuxiangyang52301@126.com (X.C.); dalingqi1999@126.com (D.Q.);

Tel.: +86-10-6829-5959 (X.C.); +86-10-6829-5858 (D.Q.);

Fax: +86-10-6829-5928 (X.C.); +86-10-6693-6619 (D.Q.).

Received: 7 May 2014; in revised form: 9 June 2014 / Accepted: 16 June 2014 / Published: 7 July 2014

\begin{abstract}
MicroRNAs (miRNAs) have essential roles in carcinogenesis and tumor progression. Here, we investigated the roles and mechanisms of miR-143 in non-small cell lung cancer (NSCLC). miR-143 was significantly decreased in NSCLC tissues and cell lines. Overexpression of miR-143 suppressed NSCLC cell proliferation, induced apoptosis, and inhibited migration and invasion in vitro. Integrated analysis identified LIM domain kinase 1 (Limk1) as a direct and functional target of miR-143. Overexpression of Limk1 attenuated the tumor suppressive effects of miR-143 in NSCLC cells. Moreover, miR-143 was inversely correlated with Limk1 expression in NSCLC tissues. Together, our results highlight the significance of miR-143 and Limk1 in the development and progression of NSCLC.
\end{abstract}


Keywords: miR-143; NSCLC; Limk1; proliferation; migration; invasion

\section{Introduction}

Non-small cell lung cancer (NSCLC), including adenocarcinoma and squamous cell carcinoma, is the leading cause of cancer deaths worldwide [1]. Despite an increasing number of studies on NSCLC genomics as well as the development of targeted therapies, the overall 5-year survival rate is only $15 \%$. This highlights the need for a better understanding of NSCLC biology to improve the prevention, diagnosis, and treatment of this cancer [2]. Recently, evidence has shown that microRNAs (miRNAs) may be involved in NSCLC pathogenesis, providing novel insights into disease biology.

miRNAs are a class of small noncoding RNAs approximately 22 nucleotides in length that suppress gene expression by post-transcriptional mechanisms. MiRNAs bind to a complementary sequence in the 3'-untranslated regions (3'-UTR) of target mRNAs, resulting in either mRNA decay or inhibition of translation [3,4]. Emerging evidence has shown that miRNAs are associated with a variety of human cancers and act as both oncogenes and tumor suppressors [5,6]. In NSCLC, multiple tumor-suppressive miRNAs have been identified, such as miR-143, miR-197, and miR-503 [7-9]. Further miRNAs have been found to promote carcinogenesis and include miR-92b, miR-96, and miR-150 [10-12]. Therefore, the aberrant expression of miRNAs could provide a novel diagnostic strategy for cancer.

miR-143 belongs to the miR-143/145 cluster, which acts as a suppressor in several tumors $[13,14]$. Recently, miRNA profiling studies have indicated that miR-143 is decreased in several tumor tissues, including NSCLC [15-17]. Furthermore, increasing evidence suggests that miR-143 plays important roles in tumor pathogenesis via the targeting of different genes $[18,19]$. In this study, we demonstrate that decreased miR-143 expression is a characteristic molecular signature in NSCLC and that miR-143 may function as a tumor suppressor by directly targeting LIM domain kinase 1 (Limk1).

\section{Results}

\section{1. miR-143 Is Downregulated in Non-Small Cell Lung Cancer (NSCLC) Tissues and Cell Lines}

To study the expression of miR-143 in NSCLC, we measured the expression of miR-143 in 24 pairs of NSCLC and matched normal lung tissues using quantitative real time PCR (qRT-PCR). The results showed that miR-143 expression was significantly down-regulated in NSCLC tissues compared with matched controls (Figure 1A). In addition, the expression of miR-143 in three NSCLC cell lines was determined, and was found to be substantially decreased compared with that in the normal cell line GNHu27 (Figure 1B). The clinicopathological features of 24 pairs of NSCLC patients are shown in Table 1, and miR-143 was correlated with the metastasis of NSCLC patients. 
Figure 1. Down-regulation of miR-143 in non-small cell lung cancer (NSCLC) tissues and cell lines. (A) The expression of miR-143 in 24 pairs of NSCLC tissues and the matched normal lung tissues (NC) was measured by quantitative real time PCR (qRT-PCR); and (B) The expression of miR-143 in normal lung epithelial cell line (GNHu27) and 3 NSCLC cell lines (A549, NCI-H23, and NCI-H358) was measured by qRT-PCR. * $p<0.05$, $* * p<0.01$ compared with control.

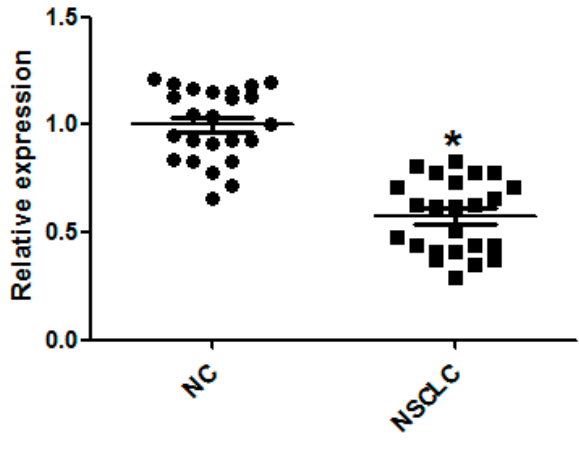

(A)

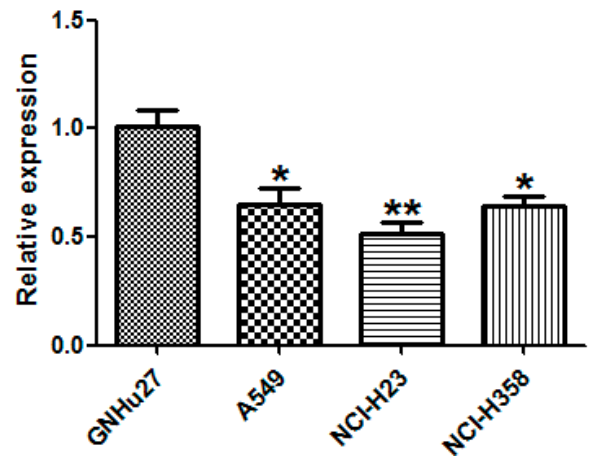

(B)

Table 1. Relationship between miR-143 and clinicopathological variables in NSCLC tissues.

\begin{tabular}{ccccc}
\hline Variable & $\boldsymbol{N}$ & Low & High & $\boldsymbol{p}$ Value \\
\hline Age (years) & 14 & & & $0.161^{\mathrm{a}}$ \\
$\geq 60$ & 10 & 6 & 6 & \\
$<60$ & 11 & & 4 & $0.542^{\mathrm{a}}$ \\
Gender & 13 & 6 & 5 & \\
Male & & 7 & 6 & $0.231^{\mathrm{a}}$ \\
Female & 14 & & & \\
Size & 10 & 4 & 6 & $0.416^{\mathrm{a}}$ \\
$>3 \mathrm{~cm}$ & & & 6 & \\
$\leq 3 \mathrm{~cm}$ & 13 & 6 & 7 & $0.214^{\mathrm{b}}$ \\
\hline Histology type & 11 & 6 & 5 & \\
Adenocarcinoma & & & & \\
Squamous cancer & 11 & 5 & 7 & $0.024^{\mathrm{a}}$ \\
\hline Histological grade & 6 & 4 & 2 & \\
I & 7 & 4 & 3 & \\
II & & & & \\
III & 13 & 9 & 4 &
\end{tabular}

${ }^{\mathrm{a}}$ Chi-square Test; ${ }^{\mathrm{b}}$ Mann-whitney Test.

\section{2. miR-143 Suppresses the Proliferation of NSCLC Cells}

To better understand the role of miR-143 in the development of NSCLC, we transfected NCI-H23 cells with miR-143 or a mimic control. Overexpression of miR-143 was confirmed by qRT-PCR (Figure 2A). The effect of miR-143 on NSCLC cell proliferation was examined using the Cell 
Counting Kit-8 (CCK-8) assay, and miR-143 was found to suppress the proliferation of NCI-H23 cells (Figure 2B). miR-143 overexpression also induced apoptosis in NCI-H23 cells (Figure 2C).

Figure 2. miR-143 suppresses the proliferation of NSCLC cells. (A) NCI-H23 cells were transfected with miR-143 or control mimic (miR-NC), and the expression of miR-143 was measured by qRT-PCR; (B) Cell viability assay (CCK-8); and (C) Apoptosis assays. Experiments were performed in triplicate. ${ }^{*} p<0.05, * * p<0.01$ compared with control.

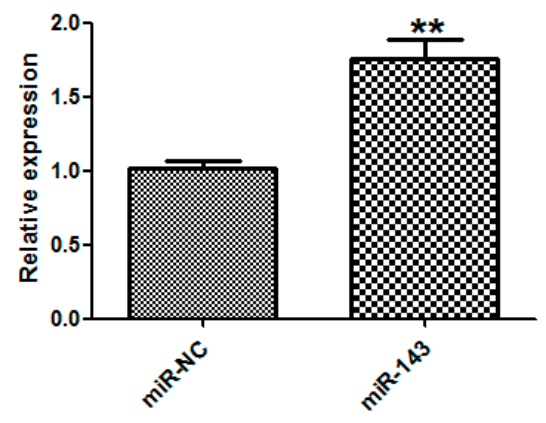

(A)

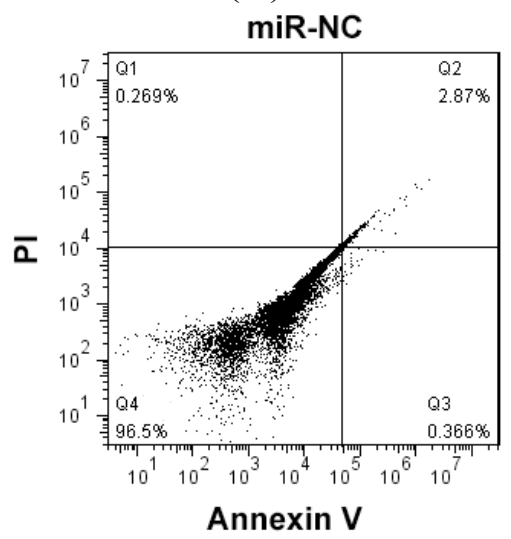

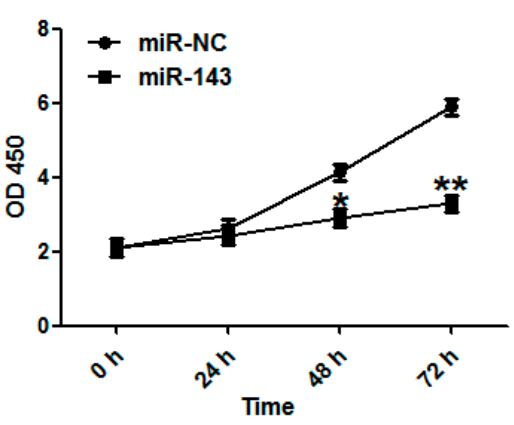

(B)

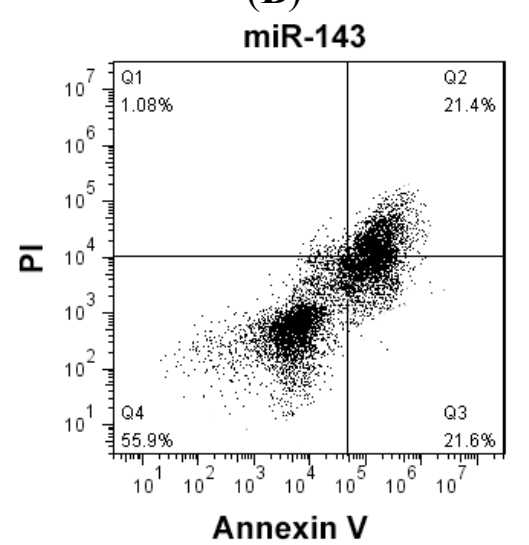

(C)

\section{3. miR-143 Suppresses Migration and Invasion of NSCLC Cells}

We further investigated whether miR-143 could inhibit migration and invasion of NSCLC. Using the Transwell migration assay, we found that overexpression of miR-143 dramatically suppressed mobility in NCI-H23 tumor cells compared with the corresponding controls (Figure 3A,B).

Figure 3. miR-143 suppresses migration and invasion of NSCLC cells. The migration (A) and invasion assays (B) of NCI-H23 cells transfected with miR-143 or miR-NC. Experiments were performed in triplicate. ${ }^{*} p<0.05, * * p<0.01$ compared with control.

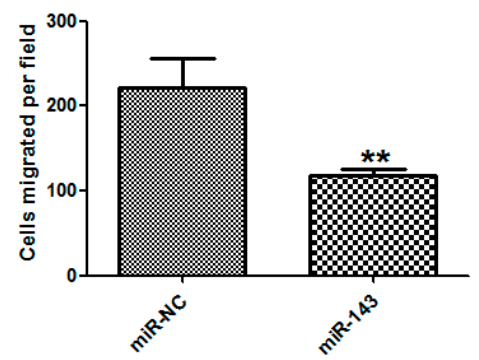

(A)

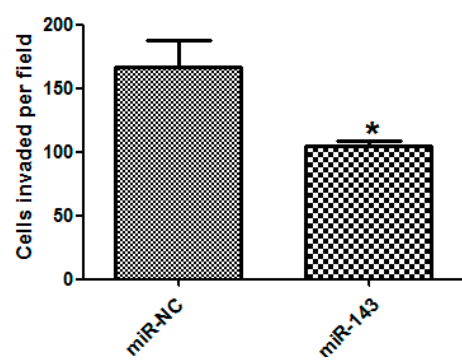

(B) 


\subsection{LIM Domain Kinase 1 (Limk1) Is a Direct Target of miR-143 in NSCLC Cells}

To investigate the downstream target of miR-143, TargetScan 6.2 was used [9]. Limk1 was predicted to be a target of miR-143 (Figure 4A). miR-143 suppressed the luciferase activity of the wild type but not the mutant 3'-UTR in HEK-293 cells (Figure 4B). Moreover, treatment with the miR-143 mimic decreased the protein level of Limk1 (Figure 4C).

Figure 4. LIM domain kinase 1 (Limk1) is a direct target of miR-143 in NSCLC cells. (A) wild type Limk1 3'-untranslated regions (3'-UTR) (WT) or Mutated Limk1 3'-UTR (Mut) of Limk1 sequence; (B) HEK-293 cells were co-transfected with miR-143/miR-NC with WT/Mut 3'-UTR of Limk1. Relative luciferase activity was assayed; and (C) NCI-H23 cells transfected with miR-143 or miR-NC, and Western blot was used to detect the protein level of Limk1. Glyceraldehyde-3-phosphate dehydrogenase (GAPDH) was used as control. Experiments were performed in triplicate. ${ }^{*} p<0.05, * * p<0.01$ compared with control.

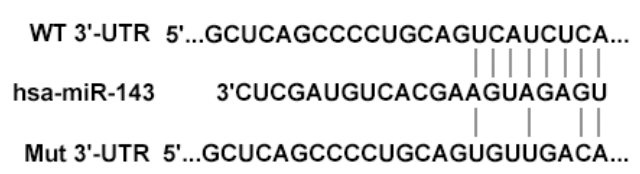

(A)

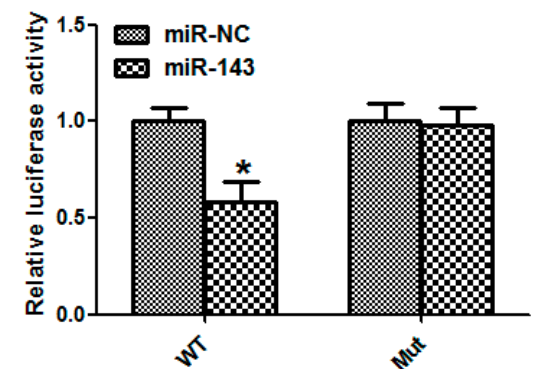

(B)

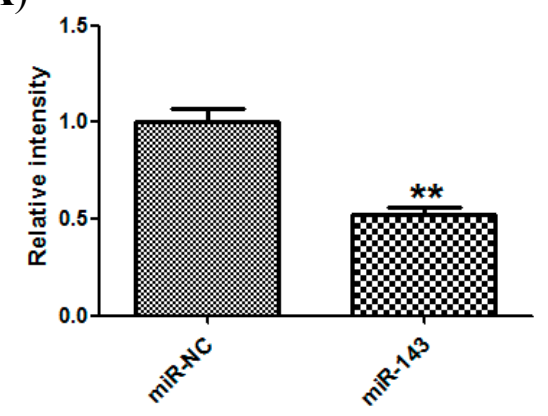

(C)

\section{5. miR-143 Suppresses NSCLC Development by Targeting Limk1}

The involvement of Limk1 in miR-143-induced suppression of NSCLC cell growth and invasion was examined using the CCK-8 assay (Figure 5A), migration (Figure 5B), and invasion (Figure 5C). In each case, Limk1 overexpression significantly attenuated the suppressive effects of miR-143 in NSCLC cells. The effect of Limk1 overexpression was confirmed by qRT-PCR (Figure 5D). 
Figure 5. miR-143 suppresses NSCLC development by targeting Limk1. (A) NCI-H23 cells were transfected with miR-143 with/without Limk1 overexpression plasmid (pcDNA-Limk1), and CCK-8 assay was performed; (B) Migration and (C) invasion assays of NCI-H23 cells; and (D) The expression of Limk1 of cells transfected with pcDNA-Limk1 or control vector was detected by qRT-PCR. The data represented three independent experiments. Experiments were performed in triplicate. $* p<0.05, * * p<0.01$ compared with control; $\# p<0.05$, \#\# $p<0.01$ compared with miR-143 transfected group.

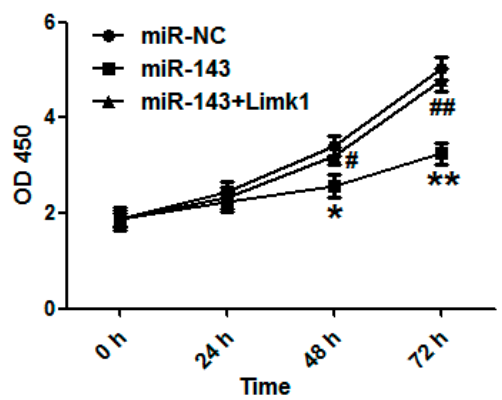

(A)

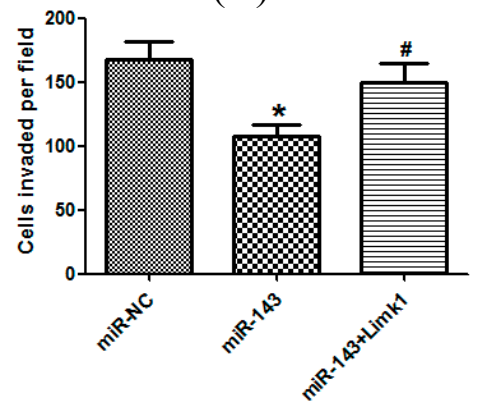

(C)

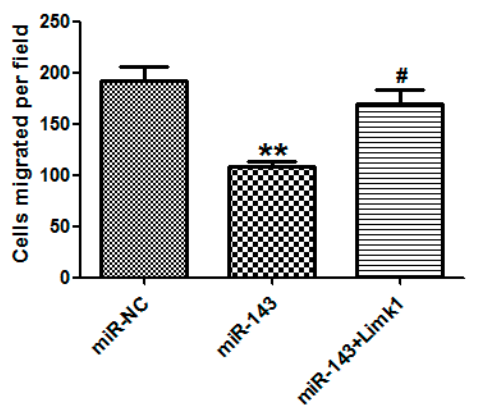

(B)

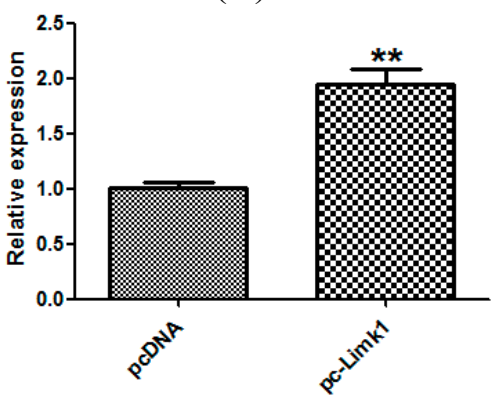

(D)

\section{6. miR-143 Expression Is Inversely Correlated with Limk1 Expression in NSCLC Tissues}

Expression of Limk1 in 24 samples of NSCLC tissue, and the corresponding normal controls, was examined by qRT-PCR. Limk1 mRNA was markedly increased in NSCLC tissues (Figure 6A). Furthermore, the level of Limk1 mRNA expression was inversely correlated with that of miR-143 NSCLC tissues (Figure 6B).

Figure 6. miR-143 is negatively correlated with Limk1 in NSCLC tissues. (A) The expression of Limk1 in 24 pairs of NSCLC tissues and the matched NC was measured by qRT-PCR; and (B) Limk1 mRNA level was inversely correlated with miR-143 level in NSCLC tissues (Spearman's correlation analysis, $r=-0.503, p=0.012$ ). ${ }^{*} p<0.05$ compared with control.

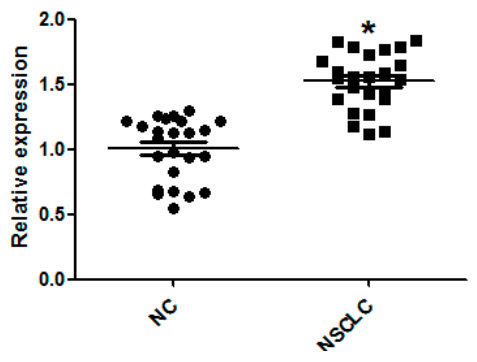

(A)

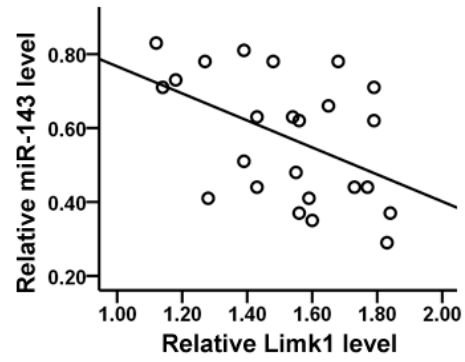

(B) 


\section{Discussion}

miRNAs have been reported to play critical roles in carcinogenesis and tumor progression [20,21]. Aberrant miRNA expression has been implicated in almost all aspects of tumor biology, including proliferation, apoptosis, migration, and invasion, and they can act as either tumor suppressors or oncogenes. Here, we focused on miR-143, which acts as a tumor suppressor in human malignancies. $\mathrm{Ng}$ et al. reported that miR-143 was significantly down-regulated in breast cancer, and that its overexpression suppressed proliferation and soft agar colony formation of breast cancer cells by down-regulating DNA methyltransferase 3A (DNMT3A) expression [15]. Zeng et al. reported that miR-143 down-regulation in peripheral blood mononuclear cells could be used as a novel diagnostic biomarker for NSCLC carcinogenesis [22]. Zhang et al. found that miR-143 regulated NSCLC cell apoptosis by inhibiting PKCE [18], and Ma et al. reported that miR-143 inhibited migration and invasion of NSCLC cells through the targeting of CD44v3 [19].

miR-143 and miR-145 are co-expressed miRNAs which form a bicistronic cluster in 5q33.1, and have been widely studied as potential tumor suppressors [23]. Viana et al. reported that miR-143 and miR-145 might be involved in the pathogenesis of benign prostatic hyperplasia via regulating target genes and proteins [24]. Kojima et al. found that in prostate cancer miR-143/145 cluster suppressed cell migration and invasion by targeting Golgi membrane protein 1 (GOLM1) [13]. Zhang et al. showed that down-regulation of miR-143 and miR-145 might be associated with overexpression of DNA methyltransferase 3B (DNMT3B) and worse prognosis in endometrioid carcinomas [25]. Cho et al. revealed that restoration of miR-145 suppressed cancer cell growth in lung adenocarcinoma patients who contained epidermal growth factor receptor (EGFR) mutation [26]. These studies suggest that miR-143 and miR-145 may co-contribute to the progression of several cancers. In NSCLC, miR-143 and miR-145 have been found to suppress the progression of NSCLC by targeting different targets $[18,19,27]$. Thus, whether miR-143 and miR-145 may co-contribute to the progression of NSCLC needs further studies.

In the present study, we showed that miR-143 was significantly down-regulated in NSCLC tissues and cell lines. Forced overexpression of miR-143 effectively suppressed NSCLC cell proliferation, enhanced apoptosis, and inhibited migration and invasion. Limk1 is a key regulator of the actin cytoskeleton, cell motility, and invasion, and is thought to be a therapeutic target for metastatic disease [28]. Limk1 is frequently overexpressed in many malignancies and functions as an important oncogene $[9,29,30]$. Downregulation of Limk1 suppressed migration of NSCLC cells and enhanced their sensitivity to chemotherapy drugs [29]. Increased Limk1 expression has been found in prostate tumor tissues, and is involved in regulating the invasiveness of prostate cancer cells [30]. Here, we demonstrate that Limk1 is a direct target of miR-143, which we confirm by luciferase activity and western blot. We found that the tumor suppressive effects of miR-143 on NSCLC cells were partially reversed by overexpression of Limk1. Finally, we have shown that Limk1 is significantly elevated in NSCLC tissues and its expression is inversely correlated with the level of miR-143 expression. Together, these data suggest that miR-143 inhibits NSCLC growth and metastasis in part through the down-regulation of Limk1.

In conclusion, the present study showed that miR-143 was significantly down-regulated in NSCLC tissues and cell lines. Forced overexpression of miR-143 inhibited tumor growth and metastasis of NSCLC cells partially through targeting Limk1. 


\section{Materials and Methods}

\subsection{Clinical Samples and Cell Lines}

Paired NSCLC and corresponding noncancerous lung tissues were obtained from 24 consecutive patients with informed consent in our department. Tissues were snap-frozen in liquid nitrogen. NSCLC cell lines A549, NCI-H23 and NCI-H358 cells, and normal GNHu27 cells were purchased from ATCC. The cell lines were maintained in RPMI-1640 medium containing $10 \% \mathrm{FBS}$ at $37{ }^{\circ} \mathrm{C}$ in a humidified atmosphere containing 5\% $\mathrm{CO}_{2}$. Transfection was performed using Lipofectamine 2000 (Invitrogen, Carlsbad, CA, USA) according to the manufacturer's protocol.

\subsection{RNA Preparation and Quantitative Real Time PCR (qRT-PCR)}

miRNAs were isolated using mir-Vana miRNA Isolation Kit from Ambion (Austin, TX, USA). Total RNAs were isolated using Trizol reagent (Invitrogen, Carlsbad, CA, USA). SYBR Green qRT-PCR was performed on ABI Stepone Plus (ABI, Foster City, CA, USA). The relative expression levels of genes were calculated using the $2^{-\Delta \Delta C t}$ method. Expression of Limk1 was normalized with Glyceraldehyde-3-phosphate dehydrogenase (GAPDH), and the level of miR-143 was normalized with U6. The primers for Limk1: Sense 5'-AGACCTCAACTCCCACAA-3' and antisense 5'-CTCAGGTGCCATCCAGT-3'.

\subsection{Plasmid Construction}

miR-143 and control mimics were obtained from RiboBio (Guangzhou, China). Limk1 cDNA was amplified and inserted into pcDNA3.1. The wild-type Limk1 3'-UTR was amplified with the following primers: Sense 5'-CCCTCGAGCCAGCAACCCTGTTCACG-3' and antisense 5'-TTGCGGCCGCC AACTGAGGCAAAGTGACAAA-3'. The PCR fragment was cloned into psiCHECK-2 vector within XhoI and NotI (Promega, Madison, WI, USA). Mutation of Limk1 3'-UTR was performed using a fast mutation kit (NEB, Ipswich, MB, Canada).

\subsection{Cell Proliferation and Apoptosis Assays}

The effect of miR-143 expression on NCI-H23 cell proliferation was assessed using CCK-8 kit (Dojindo, Kumamoto, Japan). Briefly, $5 \times 10^{3}$ transfected cells were seeded in 96-well plates and cultured for $48 \mathrm{~h}$. Exactly $10 \mu \mathrm{L}$ WST-8 was added to each well and further incubated for $1 \mathrm{~h}$ at $37{ }^{\circ} \mathrm{C}$. $\mathrm{OD}_{450}$ was measured using a microplate reader (Molecular Deviced, Sunnyvale, CA, USA).

Cells were washed with PBS and fixed with $70 \%$ ethanol. The percentage of apoptotic cells was measured by flow cytometry using the Annexin V/FITC and PI Apoptosis Detection Kit (Becton Dickinson, Cockeysville, MD, USA) according to the manufacturer instructions.

\subsection{Cell Migration and Invasion Assays}

For migration assay, $5 \times 10^{4}$ cells resuspended in serum-free RPMI-1640 after transfection were seeded in the top portion of an $8-\mu \mathrm{m}$ pore Transwell chamber (Millipore, Hayward, CA, USA). The lower chamber contained $10 \%$ FBS as a chemoattractant. Cells were incubated for $24 \mathrm{~h}$. Non-migrating cells 
on the top surface of the membrane were removed, while cells which had migrated to the bottom surface of the membrane were fixed with $95 \%$ ethanol, stained with $0.1 \%$ crystal violet, and counted. Four random fields were analyzed for each chamber.

Matrigel-coated transwell were used for invasion assay. After $48 \mathrm{~h}$, cells which had invaded to the lower surface of membrane were fixed, stained, and counted. Four random fields were analyzed for each chamber.

\subsection{Western Blot}

Forty-eight hours after transfection, cells were lysed in Radio-Immunoprecipitation Assay (RIPA) buffer (Beyotime, Shanghai, China). Equivalent amounts of lysates were loaded and separated by SDS-PAGE, and transferred onto polyvinylidene fluoride (PVDF) membranes. The membranes were blocked for $0.5 \mathrm{~h}$ at room temperature and incubated with specific primary antibodies overnight at $4{ }^{\circ} \mathrm{C}$. Membranes were further incubated with corresponding Horseradish Peroxidase (HRP)-conjugated secondary antibodies for $1 \mathrm{~h}$ at room temperature, and blots were visualized using an ECL Kit (Millipore, Hayward, CA, USA).

\subsection{Luciferase Reporter Assays}

HEK-293 cells were cultured in 24-well plate and co-transfected with $100 \mathrm{nM}$ of miR-143 or control mimics and WT or Mut using Lipofectamine 2000. Fourty-eight hours after transfection, cells were harvested, and luciferase activity was assayed using a Dual-Luciferase Reporter Assay kit (Promega, Wisconsin, WI, USA).

\subsection{Statistical Analysis}

All data were evaluated using SPSS 16. One-way ANOVA test or student's $t$ test was performed to analyze the significant differences. $p<0.05$ was considered statistically significant.

\section{Conclusions}

Our data suggest that the frequently decreased miR-143 might lead to the increased Limk1 and in turn contribute to the development of NSCLC.

\section{Acknowledgments}

We thanks the support of the First Affiliated Hospital of PLA General Hospital, and the General Hospital of People's Liberation Army.

\section{Author Contributions}

H.X., S.S., and X.C. designed the experiments. H.X., S.S., B.W., T.W., C.L., and G.L. performed the experiments. C.H. and D.Q. analyzed the data. H.X. and X.C. wrote the manuscript.

\section{Conflicts of Interest}

The authors declare no conflict of interest. 


\section{References}

1. Jemal, A.; Siegel, R.; Xu, J.; Ward, E. Cancer statistics, 2010. CA Cancer J. Clin. 2010, 60, 277-300.

2. Verdecchia, A.; Francisci, S.; Brenner, H.; Gatta, G.; Micheli, A.; Mangone, L.; Kunkler, I.; Group, E.-W. Recent cancer survival in Europe: A 2000-02 period analysis of EUROCARE-4 data. Lancet Oncol. 2007, 8, 784-796.

3. Bartel, D.P. MicroRNAs: Genomics, biogenesis, mechanism, and function. Cell 2004, 116, 281-297.

4. Bartel, D.P. MicroRNAs: Target recognition and regulatory functions. Cell 2009, 136, 215-233.

5. Zhang, X.; Nie, Y.; Li, X.; Wu, G.; Huang, Q.; Cao, J.; Du, Y.; Li, J.; Deng, R.; Huang, D. MicroRNA-181a functions as an oncomir in gastric cancer by targeting the tumour suppressor gene ATM. Pathol. Oncol. Res. 2014, 20, 381-389.

6. Yongchun, Z.; Linwei, T.; Xicai, W.; Lianhua, Y.; Guangqiang, Z.; Ming, Y.; Guangjian, L.; Yujie, L.; Yunchao, H. MicroRNA-195 inhibits non-small cell lung cancer cell proliferation, migration and invasion by targeting MYB. Cancer Lett. 2014, 347, 65-74.

7. Yang, Y.; Liu, L.; Zhang, Y.; Guan, H.; Wu, J.; Zhu, X.; Yuan, J.; Li, M. miR-503 targets PI3K p85 and IKK- $\beta$ and suppresses progression of non-small cell lung cancer. Int. J. Cancer 2014, doi:10.1002/ijc.28799. Avalable Online: onlinelibrary.wiley.com/doi/10.1002/ijc.28799/abstract (accessed on 3 March 2014).

8. Fiori, M.E.; Barbini, C.; Haas, T.L.; Marroncelli, N.; Patrizii, M.; Biffoni, M.; de Maria, R. Antitumor effect of miR-197 targeting in p53 wild-type lung cancer. Cell Death Differ. 2014, 21, 774-782.

9. Wan, L.; Zhang, L.; Fan, K.; Wang, J. miR-27b targets Limk1 to inhibit growth and invasion of NSCLC cells. Mol. Cell. Biochem. 2014, 390, 85-91.

10. Guo, H.; Li, Q.; Li, W.; Zheng, T.; Zhao, S.; Liu, Z. miR-96 downregulates RECK to promote growth and motility of non-small cell lung cancer cells. Mol. Cell. Biochem. 2014, 390, 155-160.

11. Lei, L.; Huang, Y.; Gong, W. Inhibition of miR-92b suppresses nonsmall cell lung cancer cells growth and motility by targeting RECK. Mol. Cell. Biochem. 2014, 387, 171-176.

12. Zhang, N.; Wei, X.; Xu, L. miR-150 promotes the proliferation of lung cancer cells by targeting p53. FEBS Lett. 2013, 587, 2346-2351.

13. Kojima, S.; Enokida, H.; Yoshino, H.; Itesako, T.; Chiyomaru, T.; Kinoshita, T.; Fuse, M.; Nishikawa, R.; Goto, Y.; Naya, Y. The tumor-suppressive microRNA-143/145 cluster inhibits cell migration and invasion by targeting GOLM1 in prostate cancer. J. Hum. Genet. 2014, 59, 78-87.

14. Qian, X.; Yu, J.; Yin, Y.; He, J.; Wang, L.; Li, Q.; Zhang, L.Q.; Li, C.Y.; Shi, Z.M.; Xu, Q. microRNA-143 inhibits tumor growth and angiogenesis and sensitizes chemosensitivity to oxaliplatin in colorectal cancers. Cell Cycle 2013, 12, 1385-1394.

15. Ng, E.K.; Li, R.; Shin, V.Y.; Siu, J.M.; Ma, E.S.; Kwong, A. MicroRNA-143 is down-regulated in breast cancer and regulates DNA methyltransferases 3A in breast cancer cells. Tumour Biol. 2013, $35,2591-2598$.

16. Mallick, R.; Patnaik, S.K.; Yendamuri, S. MicroRNAs and lung cancer: Biology and applications in diagnosis and prognosis. J. Carcinog. 2010, 9, 8.

17. Yanaihara, N.; Caplen, N.; Bowman, E.; Seike, M.; Kumamoto, K.; Yi, M.; Stephens, R.M.; Okamoto, A.; Yokota, J.; Tanaka, T. Unique microRNA molecular profiles in lung cancer diagnosis and prognosis. Cancer Cell 2006, 9, 189-198. 
18. Zhang, N.; Su, Y.; Xu, L. Targeting PKCepsilon by miR-143 regulates cell apoptosis in lung cancer. FEBS Lett. 2013, 587, 3661-3667.

19. Ma, Q.; Jiang, Q.; Pu, Q.; Zhang, X.; Yang, W.; Wang, Y.; Ye, S.; Wu, S.; Zhong, G.; Ren, J. microRNA-143 inhibits migration and invasion of human non-small-cell lung cancer and its relative mechanism. Int. J. Biol. Sci. 2013, 9, 680-692.

20. Wei, L.; Lian, B.; Zhang, Y.; Li, W.; Gu, J.; He, X.; Xie, L. Application of microRNA and mRNA expression profiling on prognostic biomarker discovery for hepatocellular carcinoma. BMC Gen. 2014, 15, S13.

21. Vicentini, C.; Fassan, M.; D’Angelo, E.; Corbo, V.; Silvestris, N.; Nuovo, G.J.; Scarpa, A. Clinical application of microrna testing in neuroendocrine tumors of the gastrointestinal tract. Molecules 2014, 19, 2458-2468.

22. Zeng, X.L.; Zhang, S.Y.; Zheng, J.F.; Yuan, H.; Wang, Y. Altered miR-143 and miR-150 expressions in peripheral blood mononuclear cells for diagnosis of non-small cell lung cancer. Chin. Med. J. 2013, 126, 4510-4516.

23. Takagi, T.; Iio, A.; Nakagawa, Y.; Naoe, T.; Tanigawa, N.; Akao, Y. Decreased expression of microRNA-143 and -145 in human gastric cancers. Oncology 2009, 77, 12-21.

24. Viana, N.I.; Reis, S.T.; Dip, N.G.; Morais, D.R.; Moura, C.M.; Silva, I.A.; Katz, B.; Srougi, M.; Leite, K.R.; Antunes, A.A. MicroRNAs 143 and 145 may be involved in benign prostatic hyperplasia pathogenesis through regulation of target genes and proteins. Int. J Biol. Markers [Online early access]. doi: 10.5301/jbm.5000069. Available Online: http://www.biological-markers.com /article/micrornas-143-and-145-may-be-involved-in-benign-prostatic-hyperplasia-pathogenesis-th rough-regulation-of-target-genes-and-proteins (accessed on 22 January 2014).

25. Zhang, X.; Dong, Y.; Ti, H.; Zhao, J.; Wang, Y.; Li, T.; Zhang, B. Down-regulation of miR-145 and miR-143 might be associated with DNA methyltransferase 3B overexpression and worse prognosis in endometrioid carcinomas. Hum. Pathol. 2013, 44, 2571-2580.

26. Cho, W.C.; Chow, A.S.; Au, J.S. Restoration of tumour suppressor hsa-miR-145 inhibits cancer cell growth in lung adenocarcinoma patients with epidermal growth factor receptor mutation. Eur. J. Cancer 2009, 45, 2197-2206.

27. Chen, Z.; Zeng, H.; Guo, Y.; Liu, P.; Pan, H.; Deng, A.; Hu, J. miRNA-145 inhibits non-small cell lung cancer cell proliferation by targeting c-Myc. J. Exp. Clin. Cancer Res. 2010, 29, 151.

28. Li, R.; Doherty, J.; Antonipillai, J.; Chen, S.; Devlin, M.; Visser, K.; Baell, J.; Street, I.; Anderson, R.L.; Bernard, O. LIM kinase inhibition reduces breast cancer growth and invasiveness but systemic inhibition does not reduce metastasis in mice. Clin. Exp. Metastasis 2013, 30, 483-495.

29. Chen, Q.; Jiao, D.; Hu, H.; Song, J.; Yan, J.; Wu, L.; Xu, L.Q. Down-regulation of Limk1 level inhibits migration of lung cancer cells and enhances sensitivity to chemotherapy drugs. Oncol. Res. 2013, 20, 491-498.

30. Davila, M.; Frost, A.R.; Grizzle, W.E.; Chakrabarti, R. LIM kinase 1 is essential for the invasive growth of prostate epithelial cells: Implications in prostate cancer. J. Biol. Chem. 2003, 278, 36868-36875.

(C) 2014 by the authors; licensee MDPI, Basel, Switzerland. This article is an open access article distributed under the terms and conditions of the Creative Commons Attribution license (http://creativecommons.org/licenses/by/3.0/). 